Review

\title{
Some Issues on the Foundations of Modern Cosmology, Gravitation and Quantum Physics
}

\author{
Emilio Elizalde ${ }^{1,2}$ (1) \\ 1 Consejo Superior de Investigaciones Científicas, ICE/CSIC-IEEC, Campus UAB, Carrer de Can Magrans s/n, \\ 08193 Bellaterra (Barcelona), Spain; elizalde@ieec.uab.es \\ 2 International Laboratory for Theoretical Cosmology, Tomsk State University of Control Systems and \\ Radioelectronics (TUSUR), 634050 Tomsk, Russia
}

Received: 18 September 2020; Accepted: 19 October 2020; Published: 22 October 2020

\begin{abstract}
After the first clear evidence of the recession—at very high speeds—of the spiral nebulae was announced by V.M. Slipher in 1914, as a result of his work started in 1912, it still took several decades to properly understand the phenomenon in terms of an expansion of the Universe. Some historical issues around that crucial discovery and the contemporary attempts at determining the scale of the visible Universe will be discussed. Presently, very important questions to answer are: What is the precise value of the expansion rate? What drives the acceleration of the Universe's expansion? The latter is called dark energy, but what is it actually? The possibility that this could be the result of a sort of Casimir effect at the cosmological level has not been discarded, yet. One of the main technical problems in tackling this issue is constituted by the regularization and corresponding renormalization procedures. Beautiful but rather non-trivial mathematics, involving the zeta function of pseudodifferential operators (associated with physical quantities), are key in this respect. A discussion of those items is provided here.
\end{abstract}

Keywords: universe expansion; dark energy; quantum fluctuations; zeta function regularization; models of the universe

\section{Introduction}

If I were asked to put a date to the beginning of modern cosmology I would definitely answer that this occurred in the year 1912. When we try to study the Universe as a whole, at very large scale we surely need some specific tools in order to be able to undertake such an ambitious project-namely, to accurately determine the positions and speeds of the celestial bodies. Indeed, at those enormous scales, at such extremely large distances, a whole cluster containing millions of galaxies reduces to a simple point, possessing a given mass and speed. The latter has two components: the radial velocity and its perpendicular component, projected on the celestial sphere. As I have carefully explained in previous papers [1-3] and do again in much detail in a forthcoming book [4], sufficiently reliable tools to calculate both the positions and the speeds of cosmic objects first became available in 1912, and this fact explains my choice. I will summarize the main developments around this year and the following ones in the next Section. In the trip through the paper, I will stop at three particular stages, namely, 26 April 1920, for The Great Debate on "The Scale of the Universe," August 2018, in Vienna, for the Meeting of the International Astronomical Union (IAU) and a vote on the Hubble-Lemaître law, and finally, in modernity, starting a couple of years ago, when important tensions appeared concerning the actual value of the Hubble constant.

In the paper, I will also refer to the cosmological constant problem, discussing in particular the issue of the possible contribution of quantum vacuum fluctuations to it. I will shortly address one of the main technical issues that appear in this respect, concerning the regularization and subsequent 
renormalization procedures involved. I will restrict myself to the case of the zeta function regularization of the pseudodifferential operators corresponding to physical quantities.

\section{1912: The Birth of Modern Cosmology}

There is little doubt that 1912 was the year when the main tools necessary to study the whole Universe as a dynamical system became available. Those were indeed the very first, decisive steps of cosmology as a Modern Science, which eventually led to outstanding results: the two cosmological revolutions of the 20th century. There may be some objections concerning this choice. In the same way that modern science is sometimes traced back to Galileo Galilei, for the specific case of cosmology as a science, some colleagues are ready to go as far back as Giordano Bruno. Without denying the extraordinary insight of the latter, I must argue that the tools he had at his disposal, of all kind were far from sufficient in his epoch to yield reliable numerical results as would be expected from a modern science. Additionally, it was in the year I have mentioned when this situation radically changed. Let me now explain who the main actors in the beginning of this story were, back in 1912.

The first, Henrietta Swan Leavitt, was one of the "Harvard women computers" hired by Edward Pickering, director of the Harvard College Observatory. Leavitt had received her A.B. degree from Radcliffe College in 1892, when women could not yet earn a Harvard degree. Additionally, although the Harvard College Observatory employed many female assistants, they were not allowed to operate telescopes. Their mission was to determine the brightness of the different stars in the precious collection of photographic plates of the observatory.

After several years of work collecting data, through tedious and very precise comparison of photographic plates, Leavitt discovered some 2400 variable stars. She observed a clear relationship between periods and luminosities in a sample of twenty-five of the Cepheid variables from the Small Magellanic Cloud, a satellite galaxy of our Milky Way that by then was just considered to be a nebula inside of it (as all the rest of the nebulae, including also the spiral ones). This relation was to provide the key to modern astronomical distance determinations. Leavitt plotted a graph of the luminosity versus the logarithm of the period of variability and concluded that $[5,6]$ :

"A straight line can easily be drawn between each of the two series of points corresponding to the maxima and minima, showing that there is a simple relationship between the brightness of the Cepheid variables and their periods."

Both for its simplicity and its practical importance, Leavitt's discovery is one of the most brilliant in the history of astronomy. In the words of the scientific writer Jeremy Bernstein, "Variable stars had been of interest for years, but when he was studying those plates, I doubt Pickering thought he would make a significant discovery, one that would eventually change astronomy." ${ }^{1} \mathrm{Up}$ to this point, however, it was just a law of proportionality - that is, it had still to be calibrated to determine the absolute distance. In other words, it was necessary to find the scale factor; and it turns out that the distances to the Magellanic clouds were still unknown at the time. Leavitt expressed the hope that the parallax could be measured to some of the Cepheids: The period-luminosity ratio for Cepheids, also known as Leavitt's law, made these stars the first "standard candles" in astronomy, which allowed astronomers to calculate the distances to remote galaxies; the observations of the stellar parallax are not useful at all for this purpose. Just one year after Leavitt's results, Ejnar Hertzsprung obtained the distances to several Cepheids in the Milky Way by the parallax method, and with this the calibration of the scale could be performed. With that, the distance to any other Cepheid could already be determined from then on, and Leavitt's law was firmly established. Leavitt became chief of the photographic photometry department at Harvard. Cepheids were detected in other nebulae, too, as in Andromeda.

1 Actually the document was communicated and signed by Edward Pickering, but already in its first paragraph it is indicated that it was in fact "prepared by Miss Leavitt". 
This was done by Hubble, in 1922-23, which allowed him to determine that it was outside of the Milky Way, and also, that most probably all spiral nebulae were extragalactic, located far away from our galaxy.

Leavitt's discovery changed our representation of the Universe forever. In short, her result was a precious, very powerful tool for calculating distances. Actually, her law was very much used by Edwin Hubble over the years to follow when performing such calculations, and subsequently, by several generations of astronomers, with enormous success-until other improved techniques appeared, as evolutions of the original method, which culminated in the use of supernovae SNIa as standard candles. These latter led to the discovery of the acceleration in the cosmic expansion (NP in Physics, 2011) [7,8].

Exactly the same year of 1912, in which Leavitt's fundamental work had appeared, Slipher began to work on a project that was to become extremely important, by obtaining for the first time the radial velocity of a spiral nebula, Andromeda. The idea was based on using the optical Doppler effect to determine with sufficient precision the changes in the spectral lines (Doppler shifts) towards blue or towards red. He used the 24-inch telescope at the Lowell Observatory in Arizona. The Lowell Observatory was undoubtedly at the very center of the cosmological revolution, no matter how much Edwin Hubble persisted in affirming, throughout his life, that it had been Mount Wilson exclusively.

Slipher's early research aimed to find out the rotational speeds of certain spiral galaxies, including Andromeda, and discovered-using complex photographic techniques that required exposure times as exaggerated as $80 \mathrm{~h}$-that the values of such speeds were much higher than expected. He also used spectroscopy to investigate the rotation periods of the planets and the compositions of their atmospheres. From 1912, Slipher began to apply the same method to measure the radial velocities of nebulae. A much discussed topic was by then the nature of spiral nebulae, which looked as clouds of dust or gas, but emitted a light quite similar to that of stars. Many astronomers believed they were planetary systems in formation, and only very few thought they could be star clusters located far away, outside the Milky Way; perhaps forming separate worlds, as imagined by Kant and other thinkers. To repeat, it was precisely the very same year, 1912, in which Leavitt published her most important result, when VM Slipher began his crucial project. His aim was to obtain the radial speeds of spiral nebulae from the corresponding spectra-displaced towards blue or towards red (because of the optical Doppler effect, already mentioned)—using the 24-inch telescope at the Lowell Observatory in Flagstaff, AZ, USA. It was there where, without possible argument, the first cosmological revolution of the last century had its origin.

On 17 September 1912, Slipher produced his first estimate for Andromeda (the closest nebula among the large ones). Although Slipher was already an expert, having used the spectrograph since 1909, when he turned his attention to spiral nebulae, he found that their light was so dim that he had to increase the sensitivity a lot. He reduced the size of the photographic plate to that of a thumbnail, which forced him to employ a microscope in order to analyze it. Between November and December that year, Slipher made several observations, reaching the conclusion that the Andromeda light was shifted towards the blue part of the spectrum, and more precisely, that this nebula was approaching Earth at the incredible speed of $300 \mathrm{~km} / \mathrm{s}$. This is a very high speed indeed: ten times the average velocity measured until then for all the stars in the galaxy. Slipher seriously doubted his result could be right, but Lowell thought it was a spectacular finding and encouraged him to go on and point at more nebulae. Slipher looked then at the Hat Nebula (NGC 4594), in the Virgo constellation. For this one, he got an even higher speed of $1000 \mathrm{~km} / \mathrm{s}$, and this time the nebula was clearly moving away from Earth. He went on and on, and obtained data from more spiral nebulae. By 1914 he was already able to see a clear tendency: most of the nebulae moved away and very few of them were approaching. Andromeda and a couple of other nearby nebulae were just exceptions to the general rule.

At the 17th Meeting of the American Astronomical Society, held in August 1914 at Northwestern University in Evanston, Illinois, Slipher presented the results obtained during the two previous years of very hard work, in which he had managed to measure the speeds of fifteen spiral nebulae. 
Their average speed was $400 \mathrm{~km} / \mathrm{s}$, and only three of the whole sample were approaching us; all the rest were moving away. His presentation was extremely clear and convincing to the point that his results got from the audience, according to the chronicles of the time, a huge standing ovation [9-11]. That was then very unusual in any conference of that kind, as it is now.

Slipher was the first astronomer to obtain spectra of galaxies with a signal to noise ratio good enough to determine reliable changes by Doppler shift. Additionally, his results were to shake the grounds of the by then commonly accepted model of a static universe. Slipher continued to accumulate data, and by 1917, he had the spectra for 25 spiral nebulae. Three small ones and Andromeda (all rather close objects) were approaching the Earth, while all the 21 more distant objects were again moving away, at high speeds. At this point, Slipher concluded that:

"This might suggest that the spiral nebulae are dispersing, but their distribution in the sky is not in accordance with this as they show a tendency to cluster."

The use of the word "dispersion" clearly denotes an inclination to retreat in any direction. On reading this, we may imagine that this reflection could easily have led Slipher to the idea of an expanding universe. However, we should also note that this was a very awkward concept for that epoch; in fact, it took still many years to be understood properly [12,13]. Slipher added that:

“... Our entire star system moves and takes us with it. It has long been suggested that spiral nebulae are star systems seen at great distances[...] This theory seems to me to be gaining importance in the light of the present observations."

Thus, Slipher discovered that our galaxy moved at high speed too, and that, very likely, the spiral nebulae could be analogous to our own galaxy. We should note that these thoughts were written six years before Öpik measured the actual distance to Andromeda and eight full years before Hubble found a Cepheid in this nebula, confirming in both cases that this nebula could not be, by any means, inside the Milky Way. These results, although received at first with skepticism, finally confirmed the Kantian "island universe" conjecture. For completeness, we should mention that, a few years after Slipher, Carl Wirtz also reached similar conclusions [14,15], although he was not so clear in interpreting the redshifts as Doppler shifts (velocities), for he sometimes defended the alternative interpretation (dominant in the epoch but later proven to be wrong) of them being due to the de Sitter effect.

\section{Physical Laws for the Space-Time, the Fabric of the Universe}

Quite possibly, Albert Einstein was not the first person to try to understand the nature of space and time, but no one can deny that he was probably the most successful person in this endeavor. It may be also argued that, in formulating the field equations of general relativity, he took advantage of a number of important previous results and mathematical tools of calculus and differential geometry. However, alas, the theories he eventually managed to construct, those of special and of general relativity, are extremely useful and will remain so forever. They provide a very compelling description of the origin and evolution of our universe.

Here, I will restrict myself to a rather brief discussion of some of the main issues (for more information, please see, e.g., [4]). In 1915, Einstein obtained his field equations for general relativity in their final form, and a mere one-year and a half later, in 1917, he applied them for the first time to study the Universe as a whole. At that epoch, all scholars considered that the Universe was:

1. Very small (by present scales), since it was reduced to our galaxy, the Milky Way. Thousands of other galaxies had been discovered by then, but they were all called nebulae and astronomers were absolutely convinced that they were placed inside the Milky Way. There was no question about that.

2. Static or stationary. Why? There was a strong reason for this, since under very general conditions any physical system will evolve to reach such a state, given a sufficiently large amount of time. Our Universe, for one, had had more than enough time to arrive to that state. 
3. Eternal, without a beginning or an end in time. There was not the least scientific reason that induced scientists to think otherwise.

All three of these conceptions, as reasonable and well scientifically established as they were, would radically change in few years, in what I describe in my talks and papers as the first cosmological revolution of the 20th century.

\subsection{The Great Debate of 1920}

The first of the three items above was the specific subject of a crucial one-day conference that took place exactly one hundred years ago at the time of writing, in April 1900, and which has gone into history as "The Great Debate."

Next week (as I write this) will mark a centenary since "The Great Debate, Shapley vs. Curtis," which was celebrated, on 26 April 1920, in the Baird Auditorium of the Smithsonian Museum of Natural History in Washington, D.C. The pandemic we are experiencing will prevent this anniversary from being commemorated as it should be. In 1995, on its 75th anniversary, this was done, in fact, by organizing a wonderful, very high-level meeting at the same place. Additionally, the euphoria of the moment led to the celebration of two more debates, organized soon afterwards, in 1996 and 1998 [16-20]. Today, however, that high wave has already passed, wrapped up as we are right now, astronomers and cosmologists, in finding out the true value of Hubble's "constant," the scale of the expansion of the Universe, at last, and a debate on the latter question would probably be premature by now. Anyhow, this is a very hot point, right now, and I will dedicate to it my last section of this paper.

The perspective that the past century has given us since then allows us to quote in its full value the importance and significance of that ephemeral. It was impossible then, and still is it today (after a hundred long years), to find a topic of greater relevance in cosmology than the one that gave title to the debate: "On the scale of the Universe." The aim—to clearly identify as the subject of discussion the most important one possible-was already an undoubted success.

A very precise and at the same time precious description of the state of knowledge in astronomy in 1920 can be found in the references [16-20], especially in Ref. [20] from the astronomer Virginia Trimble, from which I have extracted much of what I will mention now. It also contains careful explanations about the framework and all the political, social, economic and cultural circumstances of the time in which the event took place. The promoter of the same was the astronomer George Ellery Hale, son of the millionaire William Ellery Hale. It turns out that the father had given funds to the National Academy of Sciences (NAS), which were used, among other things, to organize annual conferences on quite different topics. Towards the end of 1919, George Hale persuaded NAS Secretary Charles Abbot to hold a one-day conference, in April 1920, in honor of his father and benefactor, William Hale. He put on the table the topic of general relativity, or alternatively, the scales of distances of the Universe. Finally, after some discussion on whether any of these topics would be sufficiently interesting for the scholarly audience, the second one was chosen.

As for the contenders, Hale initially proposed William Campbell; then director of the Lick Observatory (San Jose, California) and Harlow Shapley, Hale's associate astronomer at the Mount Wilson Observatory (Pasadena, California). They were to discuss the issue of the long-distance scale calibration, based on Cepheid stars detected in globular clusters, and other related considerations. At the last minute, it was agreed that Heber Curtis, of the Lick Observatory, would replace the director Campbell, and the title was defined exactly as: "On the Distance Scale of the Universe." Two words about the contenders. During his career as an astronomer, Curtis became very interested in the study of the Sun and in fact participated in eleven expeditions to observe eclipses, between 1900 and 1932 . However, his years at Lick had been devoted mainly to photographing spiral nebulae, and it was precisely because of this work that he was asked to face Shapley in the debate. Shapley spent much of his life as the director of different observatories, rather than as an astronomer on his feet, working with telescopes. He was Pickering's successor at the Harvard College observatory soon after the debate, and many think that his undertaking aimed at the growth and renovation of the college was 
the beginning of what this institution now represents on a global scale. In the postwar years he was president of the American Astronomical Society and also of the American Association for the Advancement of Science.

Shapley and Curtis disagreed on at least fourteen issues, which Trimble discusses in detail. They both tried to vehemently impose their views during their morning expositions, but they did so in very different styles. Direct witnesses commented that Curtis was very well organized and used slides to display graphics and other images, while Shapley limited himself to reciting his notes. Shapley defended the more extended opinion that all the Universe was limited to the Milky Way. On the contrary, Curtis expressed very serious concerns about this matter. One of the most important was that a very high number of nova stars had been found in Andromeda, as compared with other regions of the galaxy. And this clearly pointed to the possibility that this spiral nebula could be another world, a universe different from ours, although maybe quite similar to our own galaxy. Shapley countered this could not be, because one of this nova stars had been observed in Andromeda that for a few moments had eclipsed the entire nebula. If Andromeda were as far away as Curtis said, the star should have had to emit an incredible amount of energy, which was just impossible. Either way, the chronicles agree that neither of them won the debate; neither astronomer was able to convince the opponent, nor the audience. As recognized by many of the participants too, the end result of the debate and scientific discussion was a draw.

Although the participants and attendees of the debate continued to write about it with descriptions such as "the famous debate"; "memorable session"; and "anthological discussion," for several years after it took place, the fact is that the event seems to have attracted very little attention from the press, not even from the scientific one of the time. Historians have been unable to locate more than one contemporary report from December 1921. Additionally, in the obituary written after Curtis's death, the event is not mentioned anywhere. Shapley, in his 1969 autobiography, stated that he had hardly remembered the facts for a long time. The first memorial of the event dates from 1951 and it was not until 1960 that Otto Struve, writing on the 40th anniversary of the debate, referred to it as "a historical debate," although it seems that Struve could not have witnessed it in person.

Speaking of the final result, each opponent expressed at some point the opinion he believed he had won, which is perhaps not surprising, as neither of them was clearly defeated. It happened exactly like in a boxing match decided on points. The question of what the correct distance scale inside (and outside) the Milky Way is has continued to be the subject of ongoing discussion since then, and continues to be today, as I write these lines (reread, please, the beginning of this section). As I have mentioned, this is a very difficult problem in astronomy, and the Cepheids, the quintessential standard candles in that debate, were only part of the solution. The distance measurements made with them were not nearly as accurate as was believed at the time. We should recall that, using them, Hubble and Lemaître independently made mistakes of between $800 \%$ and $1000 \%$, even though no one was aware of that at the time. According to the most recent results, Shapley's galaxy was a bit too large but Curtis's one was too small (which is understandable: they both took their conclusions to opposite ends). Shapley placed the Sun much more correctly, outside the center of the Milky Way, while Curtis still placed it in the middle of it.

On the question of the existence of separate outer galaxies, or "island universes" (from Kant and other writers), Curtis made a good point on defending this hypothesis. However, he himself had to admit that it was just a "possibility" and that "data was missing," and he failed to convince his opponent or the audience. On the bright nova in Andromeda mentioned by Shapley, Kurt Lundmark was the first to raise the possibility that the phenomenon could be true, even if the star was very far away (as we all know now perfectly well, since the discovery of novae and supernovae explosions). Finally, only a couple of years later, first Öpik and then Hubble placed Andromeda undoubtedly out of the Milky Way, even out of the galaxy inflated by Shapley.

I am writing these words exactly one hundred years after "The Great Debate," and I confess that as I do so, I am getting goosebumps out of the thrill of being transported to that time, a magical, 
transcendental moment. The choice of the topic of debate was already a great success, although at the beginning it had to overcome other more popular proposals. It was then-and still is today-impossible to find a more important subject in astronomy. In fact, calculating distances using Cepheids was the origin of the first cosmological revolution of the 20th century. Not just the Earth, or the entire Solar System, but our entire galaxy—what was "the Universe," the whole world a hundred years ago-was about to become, overnight, a microscopic, insignificant spot within a new Universe, centerless and millions of times larger.

In the same way that a hundred years ago, with the knowledge they already had of the Milky Way, they could have laughed bravely at the first Universe of Anaximander, who placed the stars, together with the planets, closer to the Earth than the Sun, we could now also ridicule the official model of the Universe prevailing in 1920 and adopted by all astronomers, without exception. However, we should never do that, to scorn them: each model belongs to its time, to the data that were available in that epoch. The real lesson we should learn from here is that our magnificent current models will not be definitive either, and that knowledge about our Universe will have changed radically in one hundred years more, when the two hundredth anniversary of "The Great Debate" will be celebrated.

\subsection{An Island Universe}

In 23 November 1924, on the sixth page (top, right side) of the very famous newspaper The New York Times, this news item was published:

FINDS SPIRAL NEBULAE ARE STELLAR SYSTEMS; Dr. Hubbell Confirms View That They are 'Island Universes' Similar To Our Own.

WASHINGTON, Nov. 22. Confirmation of the view that the spiral nebulae, which appear in the heavens as whirling clouds, are in reality distant stellar systems, or "island universes", has been obtained by Dr. Edwin Hubbell of the Carnegie Institution's Mount Wilson observatory, through investigations carried out with the observatory's powerful telescopes."

The first thing that jumps out here is that Hubble's name appears misspelled, and every time. As much as I have tried, I have not been able to find out the true reason for this well known fact. If Hubble had sent a telegram to communicate the news to the newspaper, as it would be very plausible at that time, it is difficult to understand the error in the transcription of his name. If he used the phone instead, as some colleagues venture, then the error is quite understandable, since we all know how convoluted the phonetic transcription is in English (even for the natives of the language). However, I repeat that this is only a conjecture. In fact, the spelling that appeared on the NYT exists and it is widespread. Without going any further, the magic of The Web (created by CERN and MIT scientists and engineers) has allowed me, in a matter of seconds, to find out that the owners of the Hubbell mansion in Mantorville, MN, announced on Tripadvisor, are fed up with the many customers who mistakenly spell it Hubble.

Another surprising thing, and one that I will elaborate on later, is that Hubble sent his results to the press before commenting on them to anyone; that is, before discussing them with other fellow astronomers or sending them out to a specialized journal to be published. Apparently, he was absolutely convinced that they would be dismissed by his colleagues and that they would be rejected, if sent for publication, which I find quite logical, in view of the panorama of astronomy at that time. Hubble simply did not feel in any way capable of breaking the Teufelskreis created by the principal astronomers of the epoch, who defended a standard model for the Universe based on the three principles of which I have spoken before (see more details in my forthcoming book). Despite having been hired by Hale, an important astronomer, with all the pronouncements in favor, in 1919, to work at the all-important Mount Wilson Observatory in Pasadena, California, which housed then the largest telescopes of the time-as well quoted in the newspaper (the 60-inch, which had been there for some time, and a newly acquired 100-inch)_Hubble was still a relatively young astronomer. 
A year before the day of the news on the NYT, namely, towards the end of 1922, Hubble had focused the 100-inch telescope on Andromeda, looking for nova stars. In October, he took several photographic plates of what appeared to be three novae, which he marked with an N. To see if they actually were such, he examined older plates in great detail, with the purpose of finding out when each star had first appeared. Two of them disappeared, indeed, when examining old plates, but the third one remained in all of them unfailingly, although its brightness changed on the various plates: it was sometimes more intense, and other times more faint, and then it became intense again... Hubble then realized that this star was not a nova but something much more precious: a Cepheid variable star! Immediately, he crossed out, with an $\mathrm{X}$, the $\mathrm{N}$ that he had previously marked on the corresponding plate, and wrote down "Var!" next to it [21]. He had just discovered the variable star that is now called V1 and was later to became quite famous. However, calculating its variability period was not an easy task. Hubble had to examine a pile of old plates, dating back to 1909. On 23 October 1923, he was finally able to determine its period, 31.4 days; a very large value that meant the star was very distant. Using Leavitt's law-which was his main tool for calculating distances throughout his life- - he was then able to deduce that the Cepheid was at a distance of $285 \mathrm{kpc}$. That was the distance to the nebula that housed it, Andromeda. He was surprised to check that this distance was ten times more than any other one previously obtained for any celestial object, ever. This was in clear support of the hypothesis that this spiral nebula was actually beyond the Galaxy. It took him still thirteen more months to send his finding to the NYT. This was a very surprising discovery for the time and crucial for the history of cosmology. It added a very brilliant culmination to The Great Debate. Many think that, thanks to it, Curtis turned out to be, in the end, the virtual winner. It grounded the previous ideas expressed by philosophers, such as Immanuel Kant (in 1755), and some writers, Edgar Allan Poe being among them [22].

Anyhow, two years before Hubble (and only two after The Great Debate had taken place) a young but very competent Estonian astronomer, Ernst Öpik, without making any noise, had published an article in the most prestigious journal for astrophysics, the The Astrophysical Journal [23], where he had calculated that the distance to Andromeda was $450 \mathrm{kpc}$. This value is twice as close to the best one known today, of $775 \mathrm{kpc}$, compared to the result that Hubble obtained two years later! The procedure used by Öpik to calculate the distance was very different from the one employed by Hubble. It did not take any advantage of the presence of Cepheid stars, but proved to be doubly as accurate in that case. His procedure was based on the use of the rotational speeds observed around the galaxy (which inform us of its mass) and on the hypothesis that the luminosity per mass unit was equal to that of our galaxy, which allowed him to infer Andromeda's true luminosity. This method was also used by other astronomers of the time and is still in use today. Öpik was already a somewhat well-known astronomer, having been the first to calculate, six years earlier, the density of a white dwarf. In addition to these discoveries, he made several other important contributions to astrophysics, for which he received numerous distinctions. However, no one paid any attention to his extremely important result, in 1922, and only a few people among the non-astronomers even know his name today.

It is really sad and disappointing to realize how much it costs to change the descriptions of past events. And this in spite of the efforts of so many historians-and lately also of the rest of us mortals too, with the magnificent tools accessible on The Web (which I quote again)—spent in proving, day after day, the gross mistakes that are made in many of these descriptions. Öpik, like Slipher, was a sober worker, who did not spend time publicizing his results and who was not influential in science, nor did he work with the largest telescopes in the world. It is possible that Hubble did not read The Astrophysical Journal, and thus, that he was completely unaware of Öpik's result (which, on the other hand, was much better than the one he obtained). However, there is no excuse for our laziness in not renewing the history books and changing the stories from top to bottom, in view of the new evidence-watertight, perfectly proven. It seems that most chroniclers, out of laziness, do not like to spend time getting to the bottom of the facts, and simply limit themselves to copying what others have already written before. 
Anyway, even in the view of what I said, it is not easy to modify the conclusion of the previous story, actually. Let us see why. It is an incontestable historical fact that, due to his greater influence, it was Hubble who eventually changed our vision of the universe. However, this did not happen in 1924. Even being who he was, doing so would cost him a lot of work. The paradigm shift happened much more slowly than we might now think. Even after the publication of his results on Andromeda, which did not take place until 1926, or of his discovery of the redshift/distance law of recession for the nebulae (published in 1929), many of his colleagues received them with skepticism. It is only with our current eyes, with the perspective we now have of the past century, that we are able to immediately recognize the importance of the historical discovery of Öpik and Hubble. And it looks so obvious to us that we are fully unable to put ourselves in the situation of one hundred years ago, when it took the scientific community still some decades to fully realize the meaning and importance of these findings.

\subsection{The Cosmological Constant}

On 8 February 1917, Albert Einstein published an article entitled "Kosmologische Betrachtungen zur allgemeinen Relativitätstheorie" ("Cosmological Considerations on the General Theory of Relativity"), in the Journal of Physics-Mathematics Sessions of the Royal Academy of Prussia [24]. In it, he applied for the first time his brand new theory, published a year and a half before, to the whole of the Universe. He noticed at once that a stationary universe, as ours was believed to be, did not satisfy his brand new field equations. That is why he added the cosmological constant, $\lambda$, in order to solve the problem. Fourteen years later, when he was finally convinced that the universe was not stationary, but expanding, he rejected and cursed this constant, saying verbatim that, on introducing it, he had committed "the greatest blunder of my life"("die grösste Eselei meines Lebens"). He never wanted to hear about $\lambda$ again. Although, in fact, this additional constant did not hurt his equations at all, even if the universe expanded. All of his colleagues, starting with Alexander Friedmann and Georges Lemaître-who were precisely the ones who helped to prove this last fact-continued to maintain it in their solutions, the cosmological constant. Many have wondered why Einstein was so extremely radical.

Answering this question is very important, especially now that the standard cosmological model is called $\Lambda \mathrm{CDM}$, as we believe that $\Lambda$ can play a very important role in accelerating the expansion of the cosmos (the second great cosmological revolution of the 20th century). Well, it turns out that a discovery worthy of Indiana Jones himself, made in a Jerusalem archive all full of dust, and reported to me by the authors themselves (who know that lately I have been devoting some of my time to these matters), has finally allowed me to answer this question fully. I will continue with this story below.

However, let us first examine Einstein's paper. He observes in it that his theory points to the fact that the masses in the universe should have curved space so much that it should have shrunk enormously. Since the universe is still large enough and such a thing did not happen, Einstein decides to add a term with a "universal constant," which acts as "antigravity" and thus prevents the collapse of the cosmos. But let me start from the beginning, line by line. Einstein analyzes on the first page of the paper, namely, page 142 of the volume, the case for Newton's gravitation, in which exactly the same problem arises. He tries to find a solution by introducing, next to the Laplacian of the Poisson equation, a constant term, a "universal constant" $\lambda$; and argues that this idea might work. Then, he transfers it to the case of his field equations for general relativity (GR), which he obtained in his fundamental work of 1915:

$$
R_{\mu \nu}-\frac{1}{2} g_{\mu v} R=-\frac{8 \pi G}{c^{4}} T_{\mu v}
$$

Proceeding analogously, on page 151 he introduces the same type of universal constant, "eine vorläufig unbekannte universelle Konstante" ("a hitherto unknown universal constant"), into his equations of GR. With this, he gets the new equations:

$$
R_{\mu v}-\frac{1}{2} g_{\mu \nu} R-\lambda g_{\mu v}=-\frac{8 \pi G}{c^{4}} T_{\mu \nu}
$$


He argues, then, that the introduction of this additional term into his equations is compatible with all the postulates of GR, in particular with the one of covariance, according to which he constructed his fundamental theory (in fact, it is the only additional term he could introduce into it). And he argues, moreover, that as long as $\lambda$ is small enough, his new equations will continue to satisfy the critical requirement that, when applied to the domains of the solar system, they continue to give results indistinguishable from those of Newtonian physics. You can find all this out directly in Einstein's paper (provided you know some basic German). This is a good lesson to keep in mind: go, whenever possible, to the origins of the story, instead of relying on expressions that have passed from word of mouth, or transcribed from book to book, through a bunch of intermediaries. The second lesson would be: when possible, learn foreign languages.

On the last page of his article, Einstein explicitly states that he refuses to compare his model with the results of astronomical observations, limiting himself in his work to purely theoretical considerations. Many have seen a very serious mistake here, which will be reproduced in other similar attitudes throughout his life [25].

We must not be fooled by the apparent simplicity of these equations which, on the other hand, is a substantial part of their particular beauty. As an anecdote, a personal recalling of Ludwik Silberstein: During one of Arthur Eddington's lectures, he asked him: "Professor Eddington, you must be one of the only three people in the world who understand general relativity." Eddington paused, unable to answer immediately. Silberstein continued: "Please, do not be modest, Mr. Eddington!" Finally, he replied, "I am not, actually. I was just trying to guess who the third person might be." Moreover, from when Einstein paid a visit to Spain, in 1923, it is always recalled that very few people in my country were able to understand anything of what he explained, even if everyone applauded him frenetically.

The "Fifth Solvay International Conference on Electrons and Photons" was celebrated in Brussels in October 1927. It was probably the highest-level scientific conference ever held: 17 of the 29 participants featured in the meeting's famous photograph had won, or would later win, the Nobel Prize (Madame Curie did it twice). George Lemaitre was there, and they explain that in a recess of the sessions he cornered Einstein to show him his recently published article. He took the opportunity to explain to him, face to face, his discovery of a solution to Einstein's field equations for general relativity, which corresponded to an expanding universe, and that this fitted perfectly well with the latest astronomical observations. He added that he had succeeded in proving that the static solution of the universe which Einstein had obtained with the help of the cosmological constant was, in fact, unstable. The universe, in short, could not be static; it was expanding! After examining the work in some detail, the answer that Einstein gave him was as follows: he had found no error in the mathematical formulas, but the physical interpretation of those, the conclusion that the universe was expanding, did not make the slightest sense - it was "abominable" —as Lemaitre himself later detailed, in French [26]. Einstein had remembered, on seeing the equations, that Friedmann had been the first to suggest this unreal possibility five years earlier. The aggravating factor was now that he did not want to pay any attention to the clear evidence that Lemaitre provided him coming from the results of the astronomical observations, which needed to be interpreted, of course, correctly.

Yet another point. It is wrongly written in many books and articles that Hubble was the one who convinced Einstein of the universe's expansion, and that this happened when, on his tour of the United States that year, the latter visited Mount Wilson, in 1931 (there are some famous photographs of this encounter). Quite to the contrary, when perusing Einstein's notebook (Tagebuch) and also other documents of his of those days, it is possible to know with certainty that Hubble never spoke to him of the expansion of the universe. It is now known, on the contrary, that the conviction that the universe was expanding finally reached Einstein, during that same year, through conversations held with Eddington, Tolman, and de Sitter [27]. Eventually, they were able to convince him of the instability of his static model, and of the remarkable and undeniable fact that the universe was in expansion.

As we have just seen, Einstein added his "universal constant" in 1917, in his well-known attempt to obtain a model of the static universe precisely. In 1931 when he abruptly renounced 
this model: he removed the cosmological constant from his equations and embraced Friedmann's solution, but without the constant. This was 14 years after Einstein had introduced it. It was George Gamow who reported to Scientific American in 1956 that Einstein had told him, long ago, that the idea of cosmic repulsion associated with the cosmological constant had been the greatest blunder of his life ("Die grösste Eselei meines Lebens," in German). For several decades this was the only known witness to such a claim, and many historians seriously questioned it, due to the extraordinary imagination that Gamow displayed throughout his life. Recently though, thanks to the tireless work of historians of science, we now know that Einstein made a similar statement on at least two more occasions. On the one hand, John Wheeler wrote in his book Exploring Black Holes: Introduction to General Relativity (2000) that he had personally been present when Einstein said to Gamow the famous words, at the doors of the lobby of the Institute of Advanced Studies at Princeton. Another important physicist, Ralph Alpher, also testified that he heard Einstein make a very similar statement [28].

Anyhow, it is a well-proven fact that Einstein did not want to use $\lambda$ again. Not even when some colleagues suggested to him that it might be interesting to restore it, since, playing with its value, the age of the universe could be better adjusted to the results of observations on the oldest galaxies, which seemed to exceed the age of the universe itself. However, as Einstein pointed out in one of his last revisions of relativistic cosmology [29]:

"If Hubble's expansion had been discovered at the time of the creation of the general theory of relativity, the cosmological term would never have been added. It now seems much less justified to introduce such a term into field equations, as its inclusion has lost the only justification it originally had."

It is necessary to point out an important additional fact, which almost nobody emphasizes: with its position, Einstein was much more radical even than Friedmann and Lemaître, since both always included the cosmological constant term in their models of the expanding universe, although for them it was not decisive, as in Einstein's model, since for the solution of the expanding universe this additional term may play, in principle, an irrelevant role. However, if it had no reason to be there, it should not be put there; this is what Einstein stated, categorically.

At this point, we should pause for a moment to reflect on this amazing fact. Einstein was the brilliant creator of general relativity, the master of spacetime, of its contractions and dilatations, the discoverer of the possible existence of such esoteric entities as gravitational lenses and waves. Even then, it took him ten whole years (since Friedmann's solution appeared and Einstein erroneously thought there was a mistake in it), to be convinced that the universe was actually expanding. How can one explain such an attitude, such stubbornness? It may seem strange to us now, even amazing. To me, his attitude clearly illustrates a couple of points. First, it endorses my conviction that the expansion of space was, by then, an extremely revolutionary idea nobody would be ready to accept for years to come, and which some (starting with Hubble himself) could never understand, in all their lives. Lemaitre was, for a time, the only scholar who felt comfortable with the idea that physical space was actually expanding. Another question we might consider now is to what extent the latter was fortunate enough to find this correct explanation, to find the right interpretation of the astronomical results, according to the fundamental theory of Einsteinian gravitation. However, it was for him extremely difficult to convince other colleagues of the memorable discovery he had made.

\subsection{Einstein's Discarded Manuscript, Found in an Archive in Jerusalem}

However, let me get back to the point mentioned above, and finally talk about Einstein's handwritten note, from early 1931 and discovered not long ago [30]. In it, he considered a model that apparently was his last and desperate attempt to save the theory of the static universe, under the form of a steady state model. It has just four pages and was never published. It is entitled "Zum kosmologischen Problem" ("On the Cosmological Problem") and it is preserved in the archives of the Hebrew University of Jerusalem. Einstein's reasoning was this: 
"If a physically limited volume is considered, the particles of matter will be continuously leaving it. In order for the density to remain constant, new particles of matter must be produced continuously in the volume, from the space itself."

It is almost certain that this note by Einstein was never known to Hoyle, Bondi and Gold, who had exactly the same idea two decades later. With these words, Einstein describes, in essence, and in a masterful way, the same physical principle of the latter in their steady-state theory-a theory that bears some mathematical similarities to today's models of cosmic inflation and is also based on the same physical principles of general relativity.

Einstein would finally abandon his manuscript, because he found an error in the concrete way he proposed to carry out his idea: he was not able to find a suitable, specific mechanism for performing the conversion of space into matter, as he needed. He intended to make this transmutation through the cosmological constant, but realized, in a last revision he made of his note before sending it for publication, that this was not possible. The note was lost in a drawer and he never paid attention to it again. We now know that he probably missed seeing that he needed involve, at the very least, an additional term, with a matter creation field, as Hoyle, Bondi and Gold did in their model.

Thus, it is clear to me that this was the last attempt Einstein made, in early 1931, to rescue his model of a stationary universe, by using precisely the cosmological constant. I do think it was this failure that suddenly led him to renounce it in such a radical way that same year and forever after pronouncing his famous sentence.

It should be noted that in the 1940s, many physicists and astronomers still believed that the universe was unchanging, i.e., not evolving. No doubt ever arose, however, as to the validity of Hubble's empirical law, which from the outset was considered to be correct. Therefore, as we have just seen masterfully explained by Einstein himself, in order to counter the loss of matter/energy, coming from the fact that far away galaxies were moving further and further away from us, a term had to be introduced into the equations, which created matter/energy from nothing. Well, not out of nothing, but from space (as Einstein says), or if you will, out of the geometry or fluctuations of space. In short, out of the mathematical part of the GR field equations. Einstein had tried it through the cosmological constant, without success. Hoyle, Bondi and Gold achieved this through a "creation field" or "C-field," which created matter inside galaxies in distant regions of the cosmos, at a relatively slow pace, just enough to compensate for the increase in volume (equivalently, decrease in energy density) of the visible universe. In the end though, their mechanism was not very precise, and more importantly, no evidence was found then (nor has it been found so far) that this creation of matter takes place at all.

The question that needs to be clarified now is: How is it possible to generate, at least theoretically, matter and energy from a reference system, from the mathematics of spacetime?

\subsection{The Free Lunch and the Miracles of Physics}

To address this question we must turn to Einstein's GR equations. What I will now explain, in the context of the above considerations, turns out to be the same physical principle which is advocated for generating the quark-gluon plasma in current theories of cosmic inflation. Unfortunately, many scientists are unaware today that the concept of a universe with "zero total energy" or of the "free lunch" - that is, that the universe has zero total energy and maintains the balance of energy both in its formation and in its subsequent evolution-was neither discovered by Alan Guth, Andrei Linde or Alexander Vilenkin, nor by any other of the physicists dealing with inflation. All these issues were already considered and perfectly described in an absolutely masterful way in the early 1930s; for example, in the seminal book by Richard Tolman: Relativity, Thermodynamics, and Cosmology (1934). [31] There, one finds such valuable sentences like:

“... a closed universe can have total energy equal to zero. All their mass-energy is positive but their gravitational energy is negative and they can cancel each other out, which leads to a universe of zero total energy." 
This statement is now renamed, in Alan Guth's brilliant physics classes at MIT, which I have had the pleasure of attending, as the "Miracle of Physics No. 2," which reads: "The energies are not always positive, the gravitational field has negative energy." It comes after the "Miracle of Physics No. 1," which Guth explains in the same lesson, and which is in fact the one that interests us now, to begin with: "Gravity can be repulsive." Maintaining the balance of energy (or law of energy-conservation) at any time, according to general relativity, is possible as follows: one can generate a given amount of positive matter/energy as long as an equal quantity of negative spacetime pressure (also known as inflation) is available. This may correspond, for example, to an expansion of the reference system (when we use mathematical terms) or of the fabric of space (as physicists used to say, which is the same). Problem solved! This is exactly what Einstein already envisaged and very clearly expressed as a theoretical possibility, but he himself was not able to carry out in a concrete, practical way (we just saw it), in his last attempt to find use for his cosmological constant.

To better understand this most crucial point, let us consider the second Friedmann equation:

$$
\frac{\ddot{a}}{a}=-\frac{4 \pi G}{3}\left(\rho+\frac{3 p}{c^{2}}\right)+\frac{\Lambda c^{2}}{3}
$$

Without stopping to make a detailed analysis, in order to grasp the concept that concerns us, it will be enough to simply observe what follows. On the left we have the cosmic acceleration term: $a$ is the so-called scale factor, a typical length large enough (say, $100 \mathrm{Mpc}$ ) for the expansion of the universe to be perceptible; $\ddot{a}$ is its second derivative with respect to time. The minus sign, together with the density of matter $\rho$ (both in red) indicates to us that the masses of the universe slow down its expansion (Newton's law). Einstein's cosmological constant, $\Lambda$, can contribute to the acceleration of the cosmos (if positive) or to its deceleration (if negative). I leave for the end the pressure term, containing $p$; this is where the difference of GR with respect to classical mechanics lies: in this simple-looking term.

Let us understand this well: This pressure does not refer to any pressure exerted by some gas, or by a gigantic explosion that occurred somewhere in the cosmos. None of that. It is the pressure corresponding to the compression (positive pressure) or expansion (negative pressure) of space, of the very fabric of the cosmos. What really matters here is what is inside the parenthesis of the equation: matter and pressure go together; they are interchangeable. We can increase the mass of the universe (i.e., create matter and energy, which are always positive) at the expense of adding the equivalent amount of negative pressure from the fabric of space, and the equation remains the same. Negative gravity, of which Guth speaks, can be seen as an expansion of space (negative $p$ ), when compared with the ordinary gravity of the matter term, $\rho$. When there is exactly as much matter as expansion, the situation arises that the total energy of the universe is exactly zero. It is hard to go beyond what I have explained, in so few lines and at this basic a level.

To finish, I will just add a couple of things. First, the smart reader will have already noticed that I have not said anything about the precise mechanism that theories of cosmic inflation use in order to transmute negative pressure into matter (the primordial plasma), at the end of the process. I will only mention that they involve a quantum field, the inflaton, or instead quantum corrections to GR. The second is that things I have explained are not mere speculations, or non-contradictory theoretical possibilities. In fact, we have gone much further than that, to this day. To wit, analysis of the data from the most accurate astrophysical observations of WMAP, PLANCK, JLA, BAO and others, most recently from Pan-STARRS1 and the Pantheon sample [32], have clearly confirmed that the total energy of the universe is zero, within an error margin of some $0.5 \%$ or even a bit less.

\section{Zero-Point Energy and Quantum Fluctuations}

The acceleration in the expansion of the universe is a rather new concept. It was observed, for the first time, some twenty years ago. We are still lacking an accepted explanation to this phenomenon. The most natural and economic way would be to obtain it in the framework of Einstein's general relativity. That way though, we are led back to the cosmological constant term, which indeed could 
provide this acceleration, as already considered by Einstein in the first place. According to the principles of quantum physics, the fluctuations of quantum fields are unavoidable, even when the system is in the vacuum state. This is also true, in particular, for the quantum fields pervading the Universe. In the framework of Einstein's field equations, this contribution goes together with that of the cosmological constant, as discussed by Zel'dovich in a masterful way [33]. It is not just that this contribution should be there, but moreover that it is impossible for us, in principle, to get rid of it. Please observe that this argument was formulated many years in advance of the discovery of the cosmic acceleration. The cosmological constant problem, e.g., how to manage to reduce these quantum vacuum contributions to zero, was for years a separate issue [34-36].

Actually, what could have been a perfect solution magically matching the two problems $[37,38]$ turned out to be a source of new unanswered questions. On the one hand (i) the cosmological constant problem, under a new guise, since the contribution of the vacuum fluctuations of the quantum fields in the universe seems to be enormously large, under any circumstances, not just when we compare it with the value that it would be necessary to explain the universe acceleration. That adjustment requires an incredibly precise fine tuning, much more difficult to achieve than in the old situation, in which many had hoped to find a compelling reason to make it vanish.

On top of that, this quantity is not easy to calculate. Regularization and renormalization issues are involved, as will be discussed below in a bit more detail, and the range of possible final values according to the different methods and interpretations can fluctuate a lot, by many orders of magnitude; but all of them are very far from the value required. On top of that, there is also the so-called (ii) coincidence problem, which has to do with the present coincidence of the order of magnitude of the energy required to accelerate the universe expansion with the detectable matter/energy content of the universe (the first being even several times bigger). Dark energy amounts at present to some $70 \%$ of the total energy of the cosmos.

Several solutions have been proposed to solve these problems, but no one has convinced the whole community yet. Modifications of general relativity are indeed able to adjust the numbers more easily, but this is often at the price of introducing more parameters, which is never nice. Additionally, new problems appear in these theories, as the possible existence of future singularities and unwanted deviations from the standard cosmological model nowadays. Moreover, we cannot be happy with the so many possibilities, with this large number of parameters. Most of them are just effective or phenomenological models: tensor, scalar-tensor, phantom, etc. Their possible relations with fundamental theories have to be clarified.

Now on the calculations. Imposing mathematical boundary conditions on physical quantum fields—what we need to do to calculate the vacuum contribution—is also not easy at all (see, e.g., [39]). Regularization and renormalization are not able to solve the problem of the infinities which arise when putting boundaries in many cases considered and a final non-finite physical energy is obtained when the mathematical boundary conditions are imposed. Several attempts in order to solve those problems have appeared in the literature. Those of more physical nature involve setting some cut-off, in one or another way, to bring the mathematical boundary conditions to physical grounds. For instance, by observing that any physical surface will have a non-zero depth, which could be considered as a dimensional cutoff in terms of which the infinities can be regularized. Some years ago, Kurt Symanzik performed a quite rigorous study of quantum field theory in the presence of boundaries [40], which he treated at a mathematical level (no finite depth or cut-off here), using delta functions.

It is also interesting to recall here that a new approach to the problem was to insert a scalar potential of Gaussian shape acting on the surface (see for instance [41-43]). This enforced the boundary conditions on the field in question on the given surface. At a later stage the potential was left to become a delta function, and in this way the Dirichlet (or other kind) of boundary conditions (in more elaborated examples) were finally enforced. This was just at the regularization level. In all cases the method had to be completed with a corresponding quantum field theory renormalization procedure, as was also the case in Symanzik's approach. Final results agreed with [39] in various considered 
models, although they did not agree with [40]. It should be remarked that several contradictions appeared with other books and articles on the Casimir effect [44-46], where no infinities when approaching the boundaries seemed to appear. I repeat that this is not a simple issue [47-49]. In the case of gravity, the coupling of the vacuum energy is direct. In order that its influence can be manifested it is not required, in principle, that there should be any background fields or boundaries. Most reasonably, a final resolution of this issue may have to wait to the formulation of a theory of quantum gravity (or at the very least, of a reasonable approach to it).

Let me finish this section by adding that, in some cases other specific regularization methods have been successfully used to tame these divergences, such as the zeta function [50-57] and Hadamard regularization, the latter in higher post-Newtonian general relativity [58,59] and in recent variants of axiomatic and constructive quantum field theory.

\section{Zero-Point Energy}

Let us shortly recall the concept of zero-point energy. The vacuum to vacuum transition in quantum field theory is given by:

$$
\langle 0\|H\| 0\rangle,
$$

and considering the standard case of the harmonic oscillator, with spectral values $\lambda_{n}$, with normal ordering, we have

$$
H=\left(n+\frac{1}{2}\right) \lambda_{n} a_{n} a_{n}^{+}
$$

that is

$$
\langle 0|H| 0\rangle=\frac{\hbar c}{2} \sum_{n} \lambda_{n}=\frac{1}{2} \operatorname{Tr} H
$$

which gives $\infty$. Can we give a physical meaning to this quantity? The answer is affirmative, in many circumstances, as we have discussed above. In other cases, the answer is not so obvious. As explained already, regularization and renormalization procedures must be applied, e.g., cut-off, dimensional regularization, $\zeta$ function regularization techniques and operator regularization, Hadamard, etc.

Even then, after applying the regularization method and getting a finite value, has this final value a real, physical sense? Those are not simple issues that I only recall here (for more details see, e.g., $[60,61])$.

Working with zeta function regularization is not an easy job. Quite often, erroneous results have been reached, which on some occasions have been (also erroneously) attributed to a failure of the method. Time and again, it was the manner in which this rigorous method was applied that resulted in wrong answers. An important issue, often overlooked, is that this procedure is very non-linear. For instance, the zeta-trace is not a linear trace at all (the only trace in the whole class of pseudodifferential operators being the Wodzicki residue). Additionally, the zeta regularized determinant lacks the usual multiplicative property of ordinary determinants, which gives rise to the so-called non-commutative or multiplicative anomaly, which is in fact associated with the very important physical anomalies. For more details, please see references $[50-57,60,61]$.

\section{The 2018 Meeting of the IAU and the Hubble-Lemaître Law}

Two years ago, at the XXXth General Assembly of the International Astronomical Union (IAU), celebrated in Vienna (20-31 August 2018), a Resolution, tagged R4, was proposed for approval, addressing renaming the Hubble law [62]. It recommended this law to be referred to, from then on the "Hubble-Lemaitre law." The main issue was namely, that in his paper entitled "Un Univers homogene de masse constante et de rayon croissant rendant compte de la vitesse radiale des nébuleuses extra-galactiques" [63] published (in French) in 1927, Georges Lemaître rediscovered one of Friedmann's solutions, corresponding to an expanding universe. He concluded also in the paper that the spectra corresponding to distant nebulae were redshifted proportionally to their distance from the Earth. 
It was also stated in the resolution that Lemaitre made use of the tables of the redshifts as velocities and of the distances to obtain a value for the expansion rate of the Universe. A crucial point was that he did this two years before the results by Hubble appeared.

It was also argued in the presentation of the resolution that in 1950, Lemaître himself explicitly vindicated his finding, in a handwritten letter [64] where he pointed out that the value he had obtained for the expansion coefficient in his 1927 paper, of $575 \mathrm{~km} / \mathrm{sec} / \mathrm{megaparsec}$ (or of $625 \mathrm{~km} / \mathrm{sec} /$ megaparsec with a questionable statistical correction) was close to the one obtained by Hubble later, and that he was in fact pursuing a theoretical model for the Universe in accordance with the most recent astronomical results.

In the supplementary bibliography, a comment by Mario Livio published in Nature [65] was taken into account too. In the discussion, following the presentation of the resolution it was clearly established that no other cosmologist of the epoch, such as Leavitt, Slipher, Stromgren or others, had to be included in the renaming, because they had not used their data nor had they invented a new theory to discover the expansion of the universe.

It is difficult to disagree with these statements, as they are formulated. However, in previous papers I have already pointed out some crucial historical facts which had been buried or forgotten with the passage of time, and which have now been rescued from oblivion. They provide important statements directly made by some of the main actors involved, such as Hubble himself, including some revealing sentences he wrote in the last year of his life. They gave me solid reasons to suggest the proposal to include the name of Vesto Slipher in the renaming of the expansion law, to finally call it the Hubble-Lemaître-Slipher (HLS) law. I am now very conscious that this will have to wait for a future meeting and a possible new resolution [1-3].

For completeness, let me quote the final vote of Resolution R4, which was $78 \%$ in favor of the renaming, $20 \%$ against, and $2 \%$ abstentions. As was clear from the live discussion in Vienna, many of those who voted against did not do it because they actually thought that Lemaitre did not deserve to be included, but because they feared setting a precedent, as there would be so many similar cases in the history of astronomy and of science in general that should be also reconsidered, if one opened this door [62].

\section{What Is the Actual Value of the Cosmic Expansion Rate?}

This is a very hot question, which fits smoothly in the pathway marked by the previous sections. Actually, the question presently addressed may be seen as a continuation of the issue already posed at The Great Debate, one hundred years ago. The determination of the actual value of the cosmic expansion rate is a hot pending problem, which connects with the issues that started to take form one century ago, and which I have discussed in much detail at the beginning of this paper.

Indeed, the calibration of the Cepheid variables had already been an issue for Leavitt, who only obtained a proportionality law at the beginning. The first calibration, essentially the same that was used by Hubble, was later proven to be wrong for Cepheids in more distant galaxies. That led to an initial mistake in the calculation, by Hubble and others, of the cosmic expansion rate (the Hubble constant) of almost one order of magnitude. This led, in turn, to the belief that, had the universe actually had an origin, this should have happened merely 2 billion years ago, which was just impossible, since it contradicted geological data on the age of the Earth.

The Hubble constant is arguably the most important of all cosmological constants and to determine its value with good precision is correspondingly a most crucial issue. It actually turns out that the evolution of the accepted value of the Hubble constant in the past has indeed a very (over ninety years) long and extremely interesting history (see, e.g., [66,67]).

What is now under discussion, however, is another question, namely the possible evolution of the value of the expansion rate along the evolution of the universe itself. From the first calculations, this evolution seems to have been more dramatic than expected and is still, as of now, in search of a clear explanation from basic principles. I do not have much space to deal with this question (as I did 
not have so much time in my exposition at the RusGrav17 conference). It will suffice to extract some revealing results from the summaries of the main papers that prompted this discussion a few months ago, to ponder and try to understand the problem.

The title of the first in my list of relevant papers is namely, "Large Magellanic Cloud Cepheid Standards Provide a 1\% Foundation for the Determination of the Hubble Constant and Stronger Evidence for Physics Beyond $\Lambda \mathrm{CDM}$," and it is authored by Adam G. Riess, Stefano Casertano, Wenlong Yuan, Lucas M. Macri and Dan Scolnic. A summary of the main points of the abstract follows.

The authors present in this paper an improved determination of the Hubble constant $\left(H_{0}\right)$ from Hubble Space Telescope (HST) observations of 70 long-period Cepheids in the Large Magellanic Cloud, obtained with the same WFC3 photometric system used to measure Cepheids in the hosts of type Ia supernovae. They found a value of the expansion rate $H_{0}=74.22 \pm 1.82$, and combining other complementary measurements, their best estimate reads $H_{0}=74.03 \pm 1.42 \mathrm{~km} / \mathrm{s} / \mathrm{Mpc}$, including systematics, an uncertainty of $1.91-15 \%$ lower than their best previous result. As they then remarked, the difference between the value of $H_{0}$ measured locally and the value inferred from Planck $\mathrm{CMB}$ and $\Lambda C D M$ is $6.6 \pm 1.5 \mathrm{~km} / \mathrm{s} / \mathrm{Mpc}$ or 4.4 sigma ( $P=99.999 \%$ for Gaussian errors) in significance, raising therefore the discrepancy between the two values beyond a plausible level of chance. The paper was published in The Astrophysical Journal, 876:85, 1 May 2019, https:/ / doi.org/10.3847/1538-4357/ ab1422 and it has gotten over 550 citations already.

To repeat, as already pointed out by the authors, this value for the Hubble constant is in clear discrepancy with the standard one, obtained from measurements carried out by the WMAP and subsequently by the PLANCK surveyors. Let me here refer to the last one. The relevant paper is "Planck 2018 results. VI. Cosmological parameters," authored by The Planck Collaboration (which includes my former student S.R. Hildebrandt) and submitted to the arXive on 17 July 2018. It has accumulated over 3400 citations. In its abstract the authors stated that they presented results on the cosmological parameters from the Planck measurements of the CMB anisotropies corresponding to the final full-mission. Good consistency was found with the standard spatially-flat polarization, temperature and lensing, both separately and in also combination. The values obtained from the combined analysis were $\Omega_{c} h^{2}=0.120 \pm 0.001$, for the dark matter density, $\Omega_{b} h^{2}=0.0224 \pm 0.0001$ for the baryon density, $n_{s}=0.965 \pm 0.004$ for the scalar spectral index and finally $\tau=0.054 \pm 0.007$ for the optical depth. It was also quoted there that the results obtained depended only weakly on the cosmological model and that they remain stable (just with somewhat increased errors) in many of the extensions which are commonly considered. The following late-Universe parameters were inferred from those, for the base- $\Lambda$ CDM cosmology: $H_{0}=(67.4 \pm 0.5) \mathrm{km} / \mathrm{s} / \mathrm{Mpc}$ for the Hubble constant; $\Omega_{m}=0.315 \pm 0.007$ for the matter density parameter; and $\sigma_{8}=0.811 \pm 0.006$ for the amplitude of the matter fluctuations. No compelling evidence for extensions of any sort of the base- $\Lambda$ CDM model was found in this analysis.

Even it this should be rather obvious by itself, one should remark that, while these last data come form a very young universe, the ones corresponding to the first paper, and which unleashed the controversy, come from a much older universe, quite close to our epoch. A first interpretation of the difference in the results of the expansion rate is that the Hubble constant may evolve with time, with the evolution of the universe itself, although this is not the only way to explain the difference. It could be due, among other possibilities, to optic opacity of the interstellar or intergalactic media.

For completeness and comparison, let me mention another paper providing an intermediate result and relying on the tip of the red giant branch, which appeared subsequently, its title being: "The Carnegie-Chicago Hubble Program. VIII. An Independent Determination of the Hubble Constant Based on the Tip of the Red Giant Branch," authored by Wendy L. Freedman, Barry F. Madore, Dylan Hatt, Taylor J. Hoyt, In-Sung Jang, Rachael L. Beaton, Christopher R. Burns, Myung Gyoon Lee, Andrew J. Monson, Jillian R. Neeley, Mark M. Phillips, Jeffrey A. Rich and Mark Seibert. It was submitted to the arXiv on 12 July 2019, arXiv:1907.05922 [astro-ph.CO].

In this paper the authors present a new and completely independent determination of the local value of the Hubble constant based on a calibration of the tip of the red giant branch 
(TRGB) applied to type Ia supernovae (SNeIa). The value they found for the Hubble constant is $H_{0}=69.8+/-0.8(+/-1.1 \%$ stat $)+/-1.7(+/-2.4 \%$ sys $) \mathrm{km} / \mathrm{sec} / \mathrm{Mpc}$. The authors argue in the paper that the TRGB method is both precise and accurate, and that is is also independent (even if parallel to) of the Cepheid distance scale. The value obtained was seen to sit midway in the range defined by the current Hubble tension. In particular, it is seen to agree at the 1.2-sigma level with that of the Planck 2018 estimate mentioned before, and at the 1.7-sigma level with the SHoES measurement of $H_{o}$ based on the Cepheid distance scale. The TRGB distances were measured using the Advanced Camera for Surveys (ACS) imaging of galaxy halos of the deep Hubble Space Telescope (HST). The authors conclude also that, relative to Cepheid variables, there are several advantages of halo TRGB distance measurements.

This paper was published in The Astrophysical Journal 882, 34, 2019 Sep 1, https:/ / doi.org/10.3 847/1538-4357/ab2f73. Being a more recent paper, it has just reached some 170 citations up to now. Figure 17 of this last paper is actually most interesting. It shows the evolution of the Hubble constant over time and the clear discrepancy that now exists in its value obtained from the different surveys mentioned above.

I will finish this short summary by just mentioning here, in addition, a very recent article, its title being: "Measuring the Hubble constant with a sample of kilonovae," authored by Michael W. Coughlin, Sarah Antier, Tim Dietrich, Ryan J. Foley, Jack Heinzel, Mattia Bulla, Nelson Christensen, David A. Coulter, Lina Issa and Nandita Khetan. It was published very recently, on 18 August 2020, in Nature Communications 11, 4129 (2020); https: / / doi.org/10.1038/s41467-020-17998-5; arXiv:2008.07420v2 [astro-ph.HE].

In this paper, the authors argue that Kilonovae produced by the coalescence of compact binaries with at least one neutron star constitute promising standard sirens for an independent measurement of the Hubble constant $\left(H_{0}\right)$. They detected them via follow-up of gravitational-wave surveys, short gamma-ray bursts and optical surveys. They include a systematic uncertainty in the models that is as large as the statistical ones, and found as a result the following value for the Hubble constant: $H_{0}=73.8_{-5.8}^{+6.3} \mathrm{~km} / \mathrm{s} / \mathrm{Mpc}$ and $H_{0}=71.2_{-3.1}^{+3.2} \mathrm{~km} / \mathrm{s} / \mathrm{Mpc}$, which corresponds to two different kilonova models, which are consistent with the local and with the inverse-distance ladder measurements.

Summing up, this is the issue that has gained force recently: it concerns the possible evolution of the value of the expansion rate along the evolution of the universe itself. This is still, as of now, in search of a clear explanation from fundamental principles (for clear presentations of the question, see $[68,69])$.

It could eventually lead to interesting new physics. Recently, studies such as the last one above have been accumulating (see also some contributions including the author's here [70-72]), which discuss this discrepancy between modern expansion measurements on the present expansion rate of the universe and the predictions based on the CMB data measured by the PLANCK satellite-that is, on how the universe was growing over 13 billion years ago. In particular, we have addressed the issue of the possible contributions of opacity at different scales and also the all-important problem of devising a procedure for extracting information from the observational data in a model-independent way [70-72].

\section{Conclusions}

As results and final conclusions of the discussions and considerations presented in the paper, we should highlight the following. We saw in some detail that, one hundred years ago, it was not an easy task at all to manage to understand that the Universe was expanding. This was actually the first crucial cosmological discovery of the past century, which radically changed our knowledge of the Universe as a whole. We have learned that one reason (and maybe the most important) for this difficulty was that the dominant, "standard model" of the epoch had to be destroyed completely (not just reshuffled in some way). The first lesson to be drawn from there is that it is extremely difficult 
to do that, and the second is that we must be ready to do it again, when necessary. The crucial question is, has this moment arrived?

One might argue that modified or extended gravity theories (see [73-76] for some previous contributions including the author), quantum loop cosmology or quantum gravity could already do that job. However, we are still badly missing significant advances in any of those directions, not to mention in the progress of superstring theories, involving, e.g., the swampland criteria (see one preliminary attempt at a model-independent approach to this issue[77]). One may conclude that several important mysteries to be solved remain almost untouched; we cannot pretend to be even half-way to their solution.

On one hand, the evolution of the value of the expansion rate (the Hubble "constant") along the Universe's history has to be explained in a reasonable manner. On the other, the mysteries of the cosmic acceleration (dark energy) and of the nature of dark matter (of which I could not speak here) are still around. I discussed in detail the introduction by Einstein of the cosmological constant, and the problems, together with the great expectations with its interpretation as the source of dark energy (by way of the vacuum fluctuations of quantum fields). I also described the technical problems associated with these difficult calculations, which should lead us to conclude that we are probably missing something in the interpretation of the quantum fluctuations as an ordinary form of energy, in the scope of GR.

Last but not least I also discussed Einstein's unsuccessful attempt in 1931 at extracting energy from space, by means of the cosmological constant, and the subsequent one, twenty years after, of Hoyle, Bondi and Gold. The latter half succeeded with their approach, by means of the introduction of a creation field, and anticipated, with the physics of their steady state theory, the present theories of cosmic inflation.

As a general conclusion after the discussions above, today's standard theories of the origin and evolution of the cosmos may be also in need of a full revision, in view of the most recent data available (as the ones already discussed) and depending on those to be obtained in future astronomic surveys, which might confirm the deviations we start to observe from present standard physics.

Funding: This work has been partially supported by MINECO (Spain), projects FIS2016-76363-P and PID2019-104397GB-I00, and by AGAUR (Catalan Government), project 2017-SGR-247.

Acknowledgments: The author is obliged to Yurij Baryshev, Aroon Beesham, Michael Good and Sergey Sushkov for their enthusiastic comments and observations; and to Yurij also for his interesting co-authored reference [78]. The author is indebted to the anonymous referees of the paper for very detailed and useful remarks.

Conflicts of Interest: The author declares no conflict of interest.

\section{References}

1. Elizalde, E. Cosmological Constant and Dark Energy: Historical Insights. In Open Questions in Cosmology; Olmo, G.J., Ed.; InTech Publishers: Geneva, Switzerland, 2012; Chapter 1, ISBN 978-953-51-0880-1.

2. Elizalde, E. "All that matter ... in one Big Bang ...,"\& other cosmological singularities. Galaxies 2018, 6, 25.

3. Elizalde, E. Reasons in favor of a Hubble-Lemaître-Slipher's (HLS) law. Symmetry 2019, 11, 35. [CrossRef]

4. Elizalde, E. Modern Cosmology: From the Very Origins. in preparation.

5. Byers, N.; Williams, G. (Eds.) Out of the Shadows: Contributions of Twentieth-Century Women to Physics; Cambridge University Press: Cambridge, UK, 2006.

6. Lightman, A. The Discoveries: Great Breakthroughs in 20th-Century Science (Knopf, Canada, 2010). Henrietta Swan Leavitt, Wikipedia. Available online: https://en.wikipedia.org/wiki/Henrietta_Swan _Leavitt (accessed on 21 October 2020).

7. Riess, A.G.; Filippenko, A.V.; Challis, P.; Clocchiatti, A.; Diercks, A.; Garnavich, P.M.; Gillil, R.L.; Hogan, C.J.; Jha, S.; Kirshner, R.P.; et al. Observational Evidence from Supernovae for an Accelerating Universe and a Cosmological Constant. Astron. J. 1998, 116, 1009-1038. [CrossRef] 
8. Perlmutter, S.; Aldering, G.; Goldhaber, G.; Knop, R.A.; Nugent, P.; Castro, P.G.; Deustua, S.; Fabbro, S.; Goobar, A.; Groom, D.E.; et al. The Supernova Cosmology Project, Measurements of $\Omega$ and $\Lambda$ from 42 high-redshift supernovae. Astrophys. J. 1999, 517, 565-586. [CrossRef]

9. Hoyt, W.G. Vesto Melvin Slipher, Biographical Memoirs; National Academy of Sciences: Washington, DC, USA, 1980. Available online: http:/ / www.nasonline.org/publications/biographical-memoirs/memoir-pdfs/sl iphervesto.pdf (accessed on 21 October 2020).

10. Slipher, V.M. The radial velocity of the Andromeda nebula. Lowell Observ. Bull. 2012, 2, 56-57.

11. Slipher, V.M. The discovery of nebular rotation. Sci. Am. 1914, 110, 501. [CrossRef]

12. Nussbaumer, H. Slipher's Redshifts as Support for de Sitter's Model and the Discovery of the Dynamic Universe. In Proceedings of the Origins of the Expanding Universe: 1912-1932, Flagstaff, AZ, USA, 13-15 September 2012; pp. 25-38.

13. O'Raifeartaigh, C. The contribution of VM Slipher to the discovery of the expanding universe. In Proceedings of the Origins of the Expanding Universe: 1912-1932, Flagstaff, AZ, USA, 13-15 September 2012; pp. $49-63$.

14. Wirtz, C.W. Notiz zur Radialbewegung der Spiralnebel. Astron. Nachrichten 1922, 215, 451-452. [CrossRef]

15. Wirtz, C.W. Ein literarischer Hinweis zur Radialbewegung der Spiralnebel. Z. Astrophys. 1936, 11, 261.

16. Shapley, H.; Curtis, H.D. The Scale of the Universe. Bull. Natl. Res. Counc. 1921, 2, 171-217.

17. Gott, J.R., III; Juric, M.; Schlegel, D.; Hoyle, F.; Vogeley, M.; Tegmark, M.; Bahcall, N.; Brinkmann, J. A Map of the Universe. Astrophys. J. 2005, 624, 463-484. [CrossRef]

18. Shu, F. The Physical Universe, An Introduction to Astronomy; University Science Books: Mill Valley, CA, USA, 1982; p. 286.

19. Nemiroff, R.; Bonnell, J. (Org.) Great Debates in Astronomy. Available online: http:/ /apod.nasa.gov/diam ond_jubilee/debate.html (accessed on 21 October 2020).

20. Trimble, V. The 1920 Shapley-Curtis Discussion: Background, Issues, and Aftermath. Publ. Astron. Soc. Pac. 1995, 107, 1133-1144. [CrossRef]

21. Hubble's Famous M31 VAR Plate. Available online: http://obs.carnegiescience.edu/PAST/m31var (accessed on 21 October 2020).

22. Kant, I. Allgemeine Naturgeschichte und Theorie des Himmels, Nach Newtonischen Grundsatzen Abgehandelt. Johann Friederich Petersen, Königsberg und Leipzig. 1755. Available online: http: / / books.google.com/books?id=zbFDAAAAcAAJ (accessed on 21 October 2020).

23. Öpik, E. An estimate of the distance of the Andromeda Nebula. Astrophys. J. 1922, 55, 406-410; Erratum in 1923, 57, 192. [CrossRef]

24. Einstein, A. Kosmologische Betrachtungen zur allgemeinen Relativitätstheorie. Sitz. König. Preuss. Akad. 1917, VI, 142-152.

25. O'Raifeartaigh, C.; O'Keeffe, M.; McCann, B.; Nahm, W.; Mitton, S. Einstein's 1917 Static Model of the Universe: A Centennial Review. Eur. Phys. J. 2017, H42, 431-474. [CrossRef]

26. Lemaître, G. Rencontres avec Einstein. Rev. Quest. Sci. 1958, 129, 129-132.

27. Nussbaumer, H. Einstein's conversion from his static to an expanding universe. Eur. Phys. J. 2014, H39, 37-62. [CrossRef]

28. O'Raifeartaigh, C.; Mitton, S. Einstein's "Biggest Blunder"-Interrogating the Legend. arXiv 2018, arXiv:1804.06768.

29. Einstein, A. On the 'Cosmologic Problem'. Appendix I to The Meaning of Relativity, 3rd ed.; Princeton University Press: Princeton, NJ, USA, 1945; pp. 112-135.

30. O'Raifeartaigh, C.; McCann, B.; Nahm, W.; Mitton, S. Einstein's steady-state theory: An abandoned model of the cosmos. Eur. Phys. J. 2014, H39, 353-367. [CrossRef]

31. Tolman, R.C. Relativity, Thermodynamics, and Cosmology; Oxford University Press: Oxford, UK, 1934.

32. Scolnic, D.M.; Jones, D.O.; Rest, A.; Pan, Y.C.; Chornock, R.; Foley, R.J.; Huber, M.E.; Kessler, R.; Narayan, G.; Riess, A.G.; et al. The Complete Light-curve Sample of Spectroscopically Confirmed SNe Ia from Pan-STARRS1 and Cosmological Constraints from the Combined Pantheon Sample. Astrophys. J. 2018, 859, 101. [CrossRef]

33. Zeldovich, Y.B. The Cosmological constant and the theory of elementary particles. Sov. Phys. Uspekhi 1968, 11, 381-393. [CrossRef]

34. Weinberg, S. The cosmological constant problem. Rev. Mod. Phys. 1989, 61, 1. [CrossRef] 
35. Sahni V.; Starobinsky A.A. The case of a positive cosmological Lambda-term. Int. J. Mod. Phys. D 2000, 9, 373. [CrossRef]

36. Peebles, P.J.E.; Ratra, B. The cosmological constant and dark energy. Rev. Mod. Phys. 2003, 75, 559. [CrossRef]

37. Rugh, S.E.; Zinkernagel, H. The quantum vacuum and the cosmological constant problem. Stud. Hist. Philos. Sci. Part B Stud. Hist. Philos. Mod. Phys. 2002, 33, 663-705. [CrossRef]

38. Rugh, S.E.; Zinkernagel, H.; Cao, T.Y. The casimir effect and the interpretation of the vacuum. Stud. Hist. Philos. Sci. Part B Stud. Hist. Philos. Mod. Phys. 1999, 30, 111-139. [CrossRef]

39. Deutsch, D.; Candelas, P. Boundary effects in quantum field theory. Phys. Rev. D 1979, 20, 3063. [CrossRef]

40. Symanzik, K. Schrodinger Representation and Casimir Effect in Renormalizable Quantum Field Theory. Nucl. Phys. B 1981, 190, 1. [CrossRef]

41. Jaffe, R.L. Unnatural Acts: Unphysical Consequences of Imposing Boundary Conditions on Quantum Fields. AIP Conf. Proc. 2003, 687, 3-12.

42. Graham, N.; Jaffe, R.L.; Khemani, V.; Quandt, M.; Scandurra, M.; Weigel, H. Casimir Energies in Light of Quantum Field Theory. Phys. Lett. B 2003, 572, 196. [CrossRef]

43. Graham, N.; Jaffe, R.L.; Khemani, V.; Quandt, M.; Scandurra, M.; Weigel, H. Calculating Vacuum Energies in Renormalizable Quantum Field Theories: A New Approach to the Casimir Problem. Nucl. Phys. B 2002, 645, 49. [CrossRef]

44. Mostepanenko, V.M.; Trunov, N.N. The Casimir Effect and Its Application; Clarendon Press: Oxford, UK, 1997.

45. Milton, K.A. The Casimir Effect: Physical Manifestations of Zero-Point Energy; World Scientific: Singapore, 2001.

46. Bordag, M.; Mohideen, U.; Mostepanenko, V.M. New Developments in the Casimir Effect. Phys. Rep. 2001, 353, 1. [CrossRef]

47. Elizalde, E. Uses of zeta regularization in QFT with boundary conditions: A cosmo-topological Casimir effect. J. Phys. A 2006, 39, 6299. [CrossRef]

48. Elizalde, E.; Nojiri, S.; Odintsov, S.D.; Ogushi, S. Casimir effect in de Sitter and anti-de Sitter braneworlds. Phys. Rev. D 2003, 67, 063515. [CrossRef]

49. Elizalde, E.; Nojiri, S.; Odintsov, S.D.; Wang, P. Dark energy: Vacuum fluctuations, the effective phantom phase, and holography. Phys. Rev. D 2005, 71, 103504. [CrossRef]

50. Bordag, M.; Elizalde, E.; Kirsten, K. Heat kernel coefficients of the Laplace operator on the D-dimensional ball. J. Math. Phys. 1996, 37, 895. [CrossRef]

51. Bordag, M.; Elizalde, E.; Kirsten, K.; Leseduarte, S. Casimir energies for massive fields in the bag. Phys. Rev. D 1997, 56, 4896. [CrossRef]

52. Elizalde, E.; Bordag, M.; Kirsten, K. Casimir energy for a massive fermionic quantum field with a spherical boundary. J. Phys. A 1998, 31, 1743. [CrossRef]

53. Elizalde, E.; Vanzo, L.; Zerbini, S. Zeta-function regularization, the multiplicative anomaly and the Wodzicki residue. Commun. Math. Phys. 1998, 194, 613. [CrossRef]

54. Elizalde, E. Multidimensional extension of the generalized Chowla-Selberg formula. Commun. Math. Phys. 1998, 198, 83. [CrossRef]

55. Elizalde, E. Zeta functions: Formulas and applications. J. Comput. Appl. Math. 1997, 118, 125. [CrossRef]

56. Elizalde, E. Explicit zeta functions for bosonic and fermionic fields on a non-commutative toroidal spacetime. J. Phys. A 2001, 34, 3025. [CrossRef]

57. Kirsten, K.; Elizalde, E. Casimir energy of a massive field in a genus-1 surface, Phys. Lett. B 1995, $365,72$. [CrossRef]

58. Blanchet, L.; Faye, G. Hadamard regularization, J. Math. Phys. 2000, 41, 7675. [CrossRef]

59. Elizalde, E. On the issue of imposing boundary conditions on quantum fields. J. Phys. 2003, A36, L567. [CrossRef]

60. Elizalde, E. Ten Physical Applications of Spectral Zeta Functions, 2nd ed.; Lecture Notes in Physics; Springer: Berlin/Heidelberg, Germany, 2012; Volume 855.

61. Elizalde, E.; Odintsov, S.D.; Romeo, A.; Bytsenko, A.A.; Zerbini, S. Zeta Regularization Techniques with Applications; World Scientific Publishing: Singapore, 1994.

62. 2018 General Assembly, International Astronomical Union. Available online: https://www.iau.org/science/ meetings/past/general_assemblies/1180/ (accessed on 21 October 2020).

63. Lemaître, G. Un Univers homogène de masse constante et de rayon croissant rendant compte de la vitesse radiale des nébuleuses extra-galactiques. Ann. de la Société Sci. de Brux. 1927, A47, 49-59. 
64. Lemaître, G. L'expansion de l'Univers. Ann. D'Astrophys. 1950, 13, 344.

65. Livio, M. Mystery of the missing text solved. Nature 2011, 479, 171. [CrossRef]

66. Huchra, J.P. The Hubble Constant. Available online: https://www.cfa.harvard.edu/ dfabricant/huchra/h ubble/ (accessed on 21 October 2020).

67. Huchra, J.P. The Hubble Constant. Available online: https://www.cfa.harvard.edu/ dfabricant/huchra/h ubble.plot.dat (accessed on 21 October 2020).

68. Available online: https://www.nasa.gov/feature/goddard/2019/new-hubble-constant-measurement-ad ds-to-mystery-of-universe-s-expansion-rate (accessed on 21 October 2020).

69. Available online: https://www.cfa.harvard.edu/ dfabricant/huchra/hubble/ (accessed on 21 October 2020).

70. Elizalde, E.; Khurshudyan, M.; Odintsov, S.D.; Myrzakulov, R. An Analysis of the $H_{0}$ Tension Problem in a Universe with a Viscous Dark Fluid. arXiv 2006, arXiv:2006.01879.

71. Elizalde, E.; Khurshudyan, M. An Approach to the $H_{0}$ Tension Problem from Bayesian Learning and Cosmic Opacity. arXiv 2006, arXiv:2006.12913.

72. Elizalde, E.; Khurshudyan, M. Interplay between Swampland and Bayesian Machine Learning in Constraining Cosmological Models. arXiv 2007, arXiv:2007.02369.

73. Elizalde, E.; Nojiri, S.; Odintsov, S.D. Late-time cosmology in (phantom) scalar-tensor theory: Dark energy and the cosmic speed-up. Phys. Rev. D 2004, 70, 043539. [CrossRef]

74. Elizalde, E.; Odintsov, S.D.; Pozdeeva, E.O.; Vernov, S.Y. Cosmological attractor inflation from the RG-improved Higgs sector of finite gauge theory. J. Cosmol. Astropart. Phys. 2016, 1602, 025. [CrossRef]

75. Elizalde, E.; Odintsov, S.D.; Sebastiani, L.; Myrzakulov, R. Beyond-one-loop quantum gravity action yielding both inflation and late-time acceleration. Nucl. Phys. B 2017, 921, 411-435. [CrossRef]

76. Cognola, G.; Elizalde, E.; Nojiri, S.; Odintsov, S.D.; Sebastiani, L.; Zerbini, S. A class of viable modified $f(R)$ gravities describing inflation and the onset of accelerated expansion. Phys. Rev. D 2008, 77, 046009. [CrossRef]

77. Elizalde, E.; Khurshudyan, M. Swampland criteria for a dark-energy dominated universe, ensuing from Gaussian process and H(z) data analysis. Phys. Rev. D 2019, 99, 103533. [CrossRef]

78. Baryshev, Y.; Teerikorpi, P. Fundamental Questions of Practical Cosmology; Springer: Dordrecht, The Netherlands, 2012.

Publisher's Note: MDPI stays neutral with regard to jurisdictional claims in published maps and institutional affiliations.

(C) 2020 by the author. Licensee MDPI, Basel, Switzerland. This article is an open access article distributed under the terms and conditions of the Creative Commons Attribution (CC BY) license (http://creativecommons.org/licenses/by/4.0/). 